\title{
A Comparison of the Success Rate between Epidural Block and Peripheral Nerve Block in Foot Surgery
}

Yusuke Kasuya, Shota Moriwaki, Shunsaku Goto, Chiara Sonnino, Keiko Okuyama, Makoto Ozaki Department of Anaesthesiology, Tokyo Women's Medical University

\section{(O) Background and Aims}

- Epidural block and peripheral nerve block are both effective in perioperative analgesia for foot surgery. - The aim of this study was to compare the incidence of unsuccessful block between epidural block and peripheral nerve block (PNB).

\section{(O) Methods}

- The clinical outcomes of two anaesthetic techniques (GA+PNB: PNB-Group and GA+epidural block: Epi-Group) for postoperative analgesia after foot surgery in patients with rheumatoid arthritis between 2014 and 2017 were retrospectively reviewed.

- Anaesthesia was conducted at anaesthesiologist's discretion. PNB included a single-shot sciatic, a combined saphenous-sciatic, and a femoral-sciatic nerve block. For postoperative pain, supplemental analgesics were used when patients requested.

- "Unsuccessful block" was defined with the following criteria:

1) A technical failure case.

2) A case with additional analgesics required just after arousal from general anesthesia.

3) A case with rescue analgesic requested within the first 5 postoperative hours.

- Time to the first rescue analgesic after surgery and the incidence of unsuccessful block were compared between two groups.

- Survival analysis of patients without any supplemental analgesics after surgery were performed according to the Kaplan-Meier method, and the rate of unsuccessful block was calculated.

\section{(O) Results}

- Median time to the first rescue analgesic was 1681 minutes in Epi-Group and 1610 minutes in PNB-Group ( $p=0.53$ )(Fig.1).

-The incidence of unsuccessful block in Epi-Group was $36.4 \%$, and $10.8 \%$ in PNB-Group $(p=0.021)$ (Fig.2). Epi-Group patients more frequently developed postoperative early pain than PNB-Group.

\begin{tabular}{|c|c|c|c|}
\hline $\begin{array}{l}\text { Patient } \\
\text { Demographycs }\end{array}$ & $\begin{array}{l}\text { PNB-Group } \\
(\mathrm{N}=37)\end{array}$ & $\begin{array}{l}\text { Epi-Group } \\
(\mathrm{N}=33)\end{array}$ & $p$-value \\
\hline age & $64 \pm 9$ & $61 \pm 11$ & 0.21 \\
\hline $\operatorname{sex} m / f$ & $2 / 35$ & $2 / 33$ & \\
\hline Height (cm) & $154 \pm 6$ & $155 \pm 7$ & 0.53 \\
\hline Weight (kg) & $52 \pm 10$ & $51 \pm 9$ & 0.89 \\
\hline $\begin{array}{l}\text { Procedure time } \\
\text { (min) }\end{array}$ & $115 \pm 48$ & $111 \pm 35$ & 0.86 \\
\hline $\begin{array}{l}\text { Anesthesia time } \\
\text { (min) }\end{array}$ & $174 \pm 51$ & $164 \pm 37$ & 0.51 \\
\hline Fluid infusion (mL) & $1078 \pm 282$ & $1029 \pm 3$ & 0.5 \\
\hline
\end{tabular}

\section{(0) Discussion}

- Ultrasound-guided PNB technique provides a higher success rate than epidural block.

-PNB failure was more frequent in the absence of saphenous nerve block in the procedure with medial side of the forefoot. Tailor-made PNB strategy is required for achieving higher success rate.

- The percentage of rescue analgesics free patients is higher in Epi-Group after 24 hours from the surgery, which we infer is because of the difference of duration between one-shot local analgesics and continuous epidural analgesia.

- Fig 1 shows the biphasic pain rescue requirement in Epi-Group. In many cases, epidural analgesia was discontinued in the morning of the $1^{\text {st }} P O D$ (median 1249 [1121-1377] min). The balance of rehabilitation program feasibility and pain reduction is an important issue.

\section{(2) Limitation}

-The sample size is small.

- Clinical heterogeneities might be involved in this study (e.g. the wide variation in the surgical site, local anaesthetics, and different approaches).

\section{(0) Conclusion}

The incidence of unsuccessful block for foot surgery was lower in PNB than in epidural block.

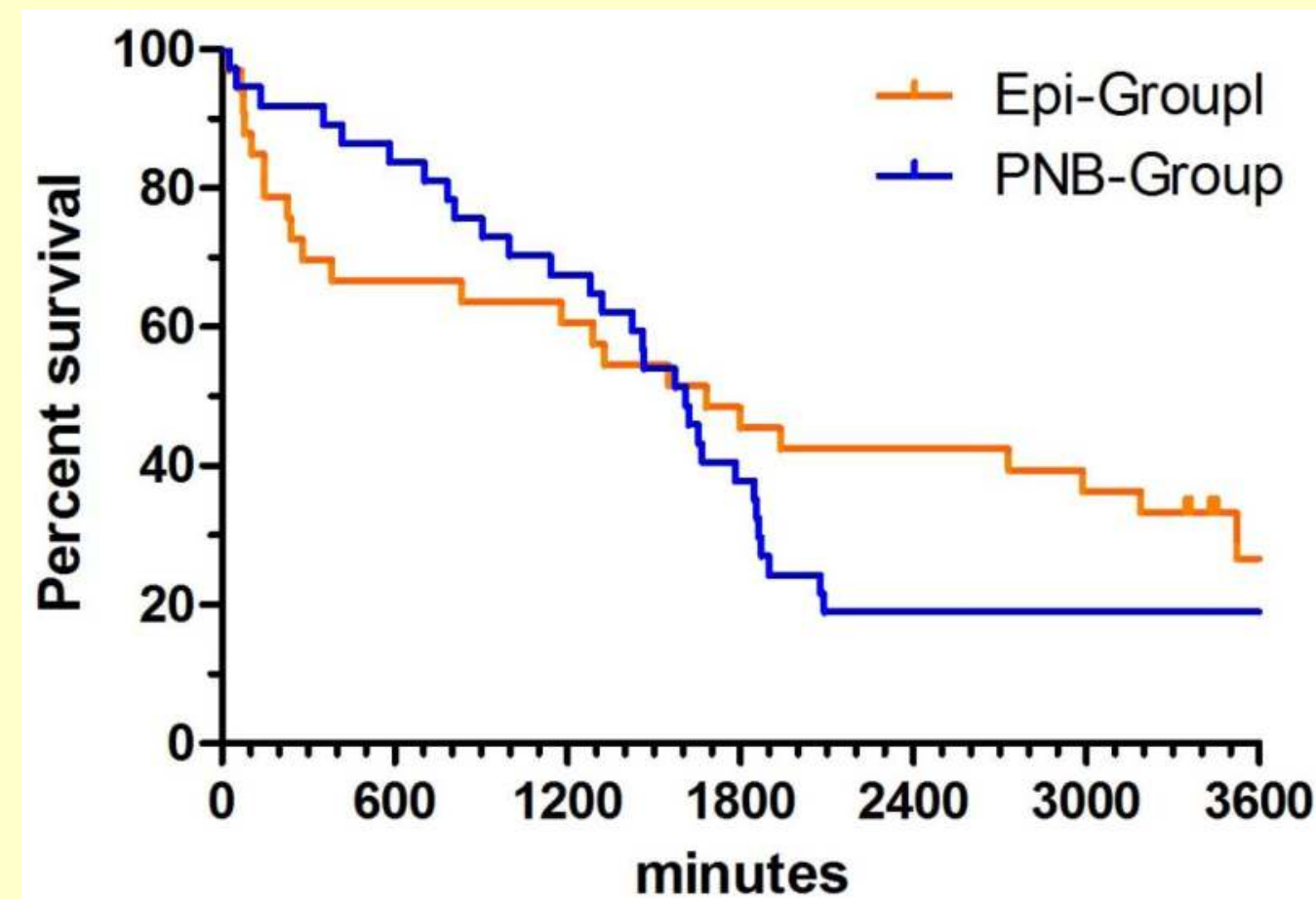

Fig.1 Kaplan-Meier curves of percentage of patients without any supplemental analgesics after surgery in Epi-Group and PNB-Group

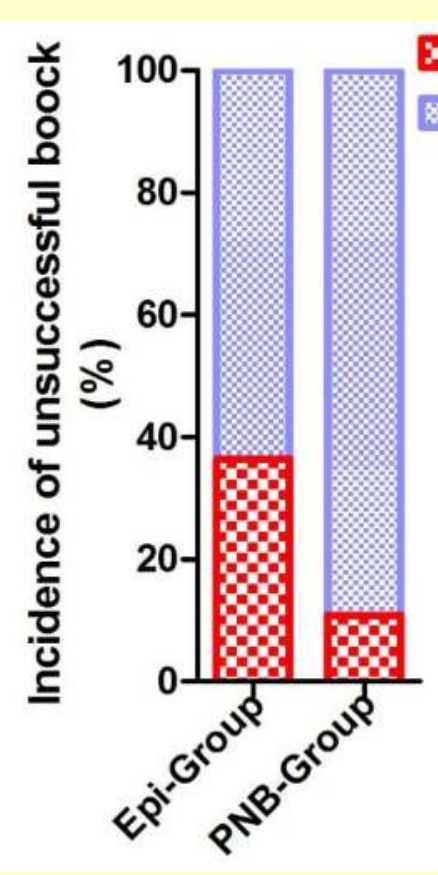

Fig.2 Unsuccessful block Successful block
Reason for unsuccessful block Technical Failure Pain at the arousal of anesthesia

Pain rescue within 5 hours postoperatively

Total Failure case *Two cases were unable to use epicatheter because of blood aspiration.

The rate of unsuccessful blook in Epi-Group and PNB-Group 